\title{
Dysphagie causée par un anévrisme aortique et une dilatation atriale gauche chez un homme de 89 ans
}

\author{
Ziran Meng MD, Michael Pereira MD, Aditya Sharma MD
}

— Citation : CMAJ 2020 November 23;192:E1539. doi : 10.1503/cmaj.200427-f

Voir la version anglaise de l'article ici : www.cmaj.ca/lookup/doi/10.1503/cmaj.200427

$\mathbf{U}$

n homme de 89 ans chez qui on avait réparé un anévrisme aortique abdominal a été hospitalisé après avoir perdu $9 \mathrm{~kg}$ en 6 mois en raison d'une dysphagie évolutive gênant la consommation d'aliments solides et liquides. L'œsophagogastroduodénoscopie a révélé une masse œsophagienne atypique (figure 1A); soupçonnant la présence d'ectasies vasculaires, nous avons toutefois décidé de ne pas faire de biopsie de la lésion. La radiographie pulmonaire a montré une cardiomégalie et une dilatation atriale gauche (figure 1B). La tomodensitométrie thoracique n'a pas montré de lésions œsophagiennes bien délimitées; elle a cependant révélé un anévrisme thoracoabdominal sinueux de $6,7 \mathrm{~cm}$ au niveau du hiatus et de $9,5 \mathrm{~cm}$ au niveau du tronc cœliaque. Elle a également permis de détecter la présence d'une dilatation atriale gauche mesurant $7,8 \mathrm{~cm}$ à son paroxysme (figure $1 \mathrm{C}$ et annexe 1 , accessible en anglais au www.cmaj.ca/lookup/doi/10.1503/cmaj.200427/ tab-related-content). Par la suite, une radiographie au baryum de l'œsophage a révélé une rétention substantielle de l'agent de contraste au niveau du sphincter œsophagien inférieur, attribuable à une compression extrinsèque et à une dysmotilité liée à l'âge (figure 1D). Notre patient a opté pour une prise en charge prudente, la réparation chirurgicale comportant des risques importants. Nous avons consulté les services de nutrition, qui ont recommandé une diète d'aliments mous pour aider le patient à composer avec sa dysphagie.

Le terme "dysphagia aortica » englobe les difficultés de déglutition dues à une compression extrinsèque de l'œsophage par une dilatation aortique. La « dysphagia megalatriensis » est quant à elle causée par une compression externe de l'œsophage découlant une dilatation atriale gauche. Ces 2 types de dysphagie sont peu courants, et leur présence com-
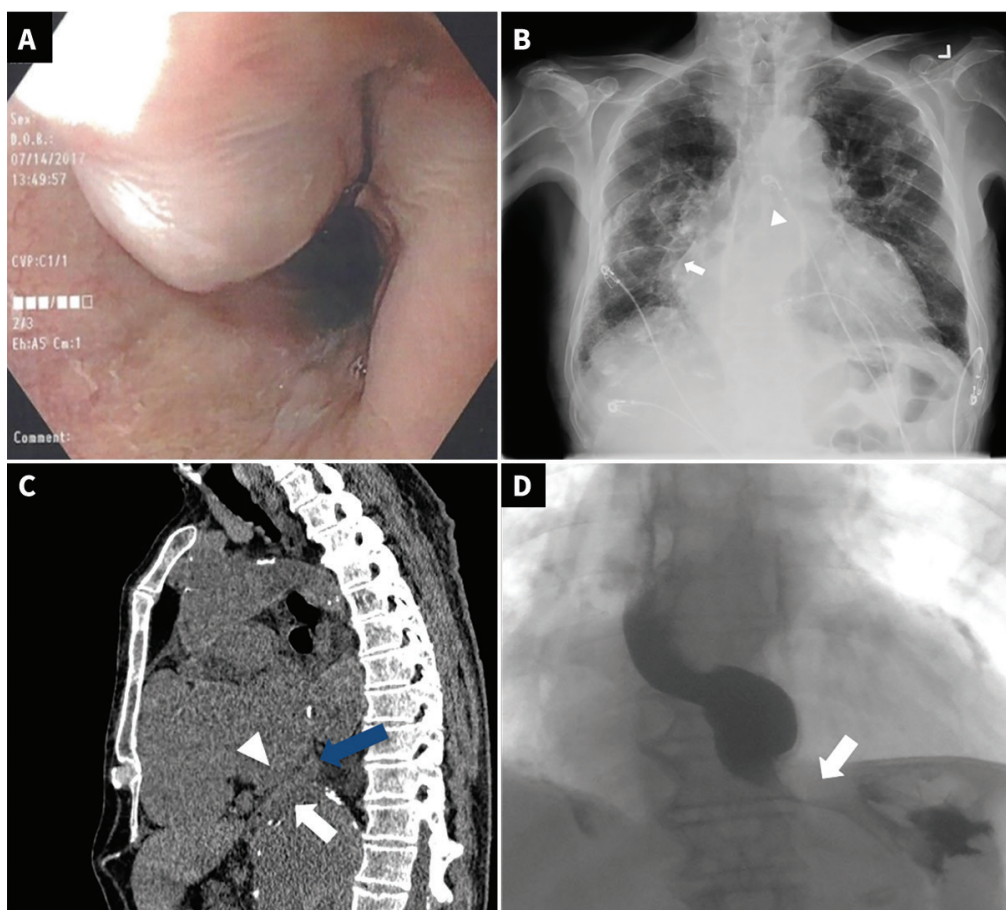

D

Figure 1 : Résultats des tests d'un homme de 89 ans présentant une dysphagie évolutive et une perte de poids. A) Esophagogastroduodénoscopie montrant une compression externe de la lumière de l'œsophage. B) Radiographie pulmonaire postéro-antérieure avec signes de dilatation atriale gauche (aspect de double contour [flèche blanche] et élargissement de l'angle de la carène [pointe de la flèche]). C) Image en plan sagittal captée par tomodensitométrie montrant une compression externe de l'œsophage (flèche bleue) par l'atrium gauche (pointe de la flèche) et l'aorte (flèche blanche). D) Radiographie au baryum de l'œsophage montrant une compression extrinsèque de la partie inférieure de l'œsophage (flèche blanche). 
sous-jacent permettrait d'atténuer les symptômes ${ }^{1,3,4}$. Chez les patients qui ne peuvent pas subir d'intervention chirurgicale, on peut envisager la pose d'une sonde d'alimentation percutanée ou l'adoption d'un régime d'aliments mous, hachés ou émincés.

\section{Références}

1. Choi SH, Yang G, Gagnon J. Dysphagia aortica secondary to thoracoabdominal aortic aneurysm resolved after endograft placement. J Vasc Surg Cases Innov Tech 2019;5:501-5.

2. Wilkinson JM, Euinton $\mathrm{H}, \mathrm{Smith} \mathrm{L}$, et al. Diagnostic dilemmas in dysphagia aortica. Eur J Cardiothorac Surg 1997;11:222-7.

3. Siddiqui J, Hughes F. Dysphagia due to thoracic aortic aneurysm, relieved by thoracic endovascular aneurysm repair: a case report and review of the literature. BMJ Case Rep 2011;2011:bcr0920114793.

4. Zia HA, Madhoun M. Cardiogenic dysphagia by dysphagia megalatriensis and aortica. Clin Gastroenterol Hepatol 2020;18:e21.

5. Kim JH, Jang S, Kim D, et al. A patient with dysphagia due to an aortic aneurysm. Korean Circ J 2009;39:258-60.

Intérêts concurrents : Aucun déclaré.

Cet article a été révisé par des pairs.

Les auteurs ont obtenu le consentement du plus proche parent.

Affiliations : Départements de médecine interne (Meng, Sharma) et de radiologie (Pereira), Université du Manitoba, Winnipeg, Man.

Propriété intellectuelle du contenu : Il s'agit d'un article en libre accès distribué conformément aux modalités de la licence Creative Commons Attribution (CC BY-NC-ND 4.0), qui permet l'utilisation, la diffusion et la reproduction dans tout médium à la condition que la publication originale soit adéquatement citée, que l'utilisation se fasse à des fins non commerciales (c.-à-d., recherche ou éducation) et qu'aucune modification ni adaptation n'y soit apportée. Voir : https:// creativecommons.org/licenses/by-nc-nd/4.0/deed.fr

Correspondance : Ziran Meng, mengz2000@gmail.com 